\title{
Towards Induction Mapping of the 3D Spin Texture of Skyrmions
}

S. Schneider ${ }^{1,2}$, D. Wolf ${ }^{1}$, M. J. Stolt ${ }^{3}$, S. Jin ${ }^{3}$, D. Pohl ${ }^{1,4}$, B. Rellinghaus ${ }^{1,4}$, M. Schmidt ${ }^{5}$, B. Büchner ${ }^{1}$, S. T. B. Goennenwein ${ }^{2,6}$, K. Nielsch ${ }^{1,7}$, A. Lubk ${ }^{1}$

1. IFW Dresden, Dresden, Germany.

2. TU Dresden, Institut für Festkörper- und Materialphysik, Dresden, Germany.

3. University of Wisconsin-Madison, Department of Chemistry, Madison, WI, United States.

4. TU Dresden, Dresden Center for Nanoanalysis, Dresden, Germany.

5. Max Planck Institute for Chemical Physics of Solids, Dresden, Germany.

6. TU Dresden, Center for Transport and Devices of Emergent Materials, Dresden, Germany.

7. TU Dresden, Institute of Materials Science, Dresden, Germany.

Skyrmions [1] are topologically non-trivial vortex-like spin textures, anticipated for application in spintronic technologies, in next generation magnetic data processing and storage due to their facile manipulation by spin-polarized currents of very low magnitude [2,3]. Unfortunately, little is known about the 3D structure of skyrmions [4, 5], ubiquitous in thin film technology. Here, we bridge that gap by combining the concept of the transport of intensity equation (TIE) [6], focal series in-line electron holography (EH), and off-axis EH [7] to quantitatively reconstruct the projected magnetic field pertaining to both the helical and the skyrmion lattice phase in single crystal nanoparticles of the isotropic chiral magnet $\mathrm{Fe}_{0.95} \mathrm{Co}_{0.05} \mathrm{Ge}$.

The skyrmion phase in $\mathrm{Fe}_{0.95} \mathrm{Co}_{0.05} \mathrm{Ge}$ particles (Fig. 1 (b)) was investigated using a double corrected FEI Titan ${ }^{3}$ 80-300 microscope operated in image corrected Lorentz mode. A focal series of Lorentz TEM (L-TEM) images of a single isolated nanoplatelett oriented along the [001] zone axis (Fig. 1 (a)) was recorded. Reconstruction of the phase of the electron wave and thereby of the magnetic induction was obtained with the help of a modified Gerchberg-Saxton type algorithm. To supplement the focal series reconstructions from large field of views, smaller areas of the identical nanoplatelett were investigated by off-axis EH. A direct tomographic investigation of the 3D structure of the skyrmionic lattice is currently experimentally unfeasible, because this would require an externally applied out-ofplane magnetic field to be tilted with the sample.

Thus, indirect experimental evidence for the 3D structure of the skyrmionic lattice may currently only be inferred from a quantitative analysis of the projected magnetic induction in the sample conducted with the help of EH. Fig. 2 (a) depicts an underfocused L-TEM micrograph showing the hexagonal that reveals the skyrmion lattice. The image is one out of 21 of the focal series used for an in-line holography reconstruction of the object exit wave in amplitude and phase. Figs. $2(b, c)$ show magnetic induction maps $\bar{B}_{\perp}(x, y)$ in cylindrical coordinate representation visualizing the spin texture of the skyrmions by $\bar{B}_{\phi}(x, y)$ (Fig. 2(b)) and their donut-shaped magnitude by $\bar{B}_{r}(x, y)$ (Fig. 2 (c)). Likewise, we observed magnetic induction maps (Figs. 2 (e, f)) from a phase image reconstructed by off-axis EH (Fig. 2(d)) on the same $\mathrm{Fe}_{0.95} \mathrm{Co}_{0.05} \mathrm{Ge}$ nanoplatelett. Most strikingly, we consistently observe a reduction of the projected in-plane B-fields $\left(\bar{B}_{\max }=(0.2 \ldots 0.3) T\right)$ as compared to those of a homogeneous skyrmion throughout the film thickness. Two alternative models for the 3D structure of skyrmions are thus derived. 


\section{References:}

[1] A. N. Bogdanov and A. Hubert, Journal of Magnetism and Magnetic Materials 138 (1994), p, 255.

[2] N. Nagaosa and Y. Tokura, Nature Nanotechnology 8 (2013), p. 899.

[3] N. Kanazawa et al., „Noncentrosymmetric Magnets Hosting Magnetic Skyrmions“ (2017).

[4] F. N. Rybakov et al., Physical Review B - Condensed Matter and Materials Physics 87 (2013), p. 094424.

[5] H. S. Park et al., Nature Nanotechnology 9 (2014), p. 337.

[6] A. Lubk et al., Physical Review Letters 111 (2013), p. 173902.

[7] H. Lichte et al., Ultramicroscopy 134 (2013), p. 126.
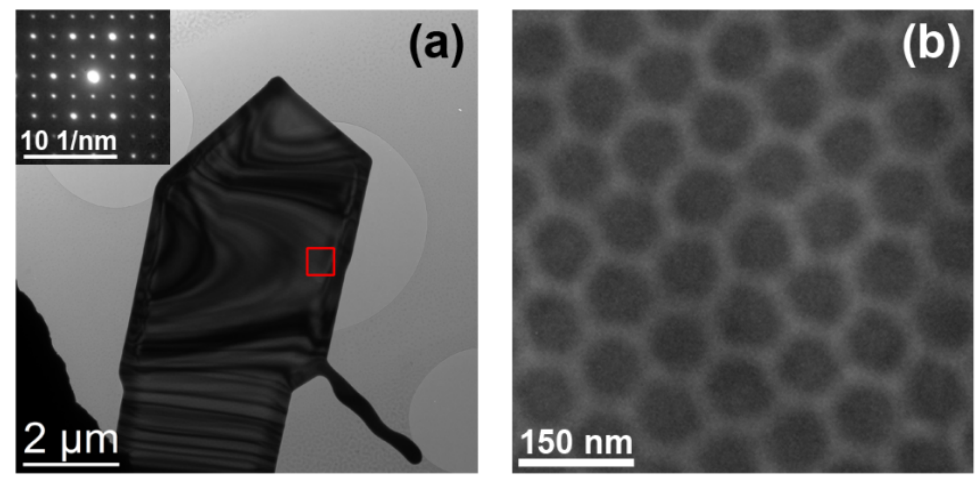

Figure 1. (a) TEM image of a $\mathrm{Fe}_{0.95} \mathrm{Co}_{0.05} \mathrm{Ge}$ nanoplatelett in [001] with the diffraction pattern in the inset. (b) Skyrmion lattice as observed within the marked area in panel (a).

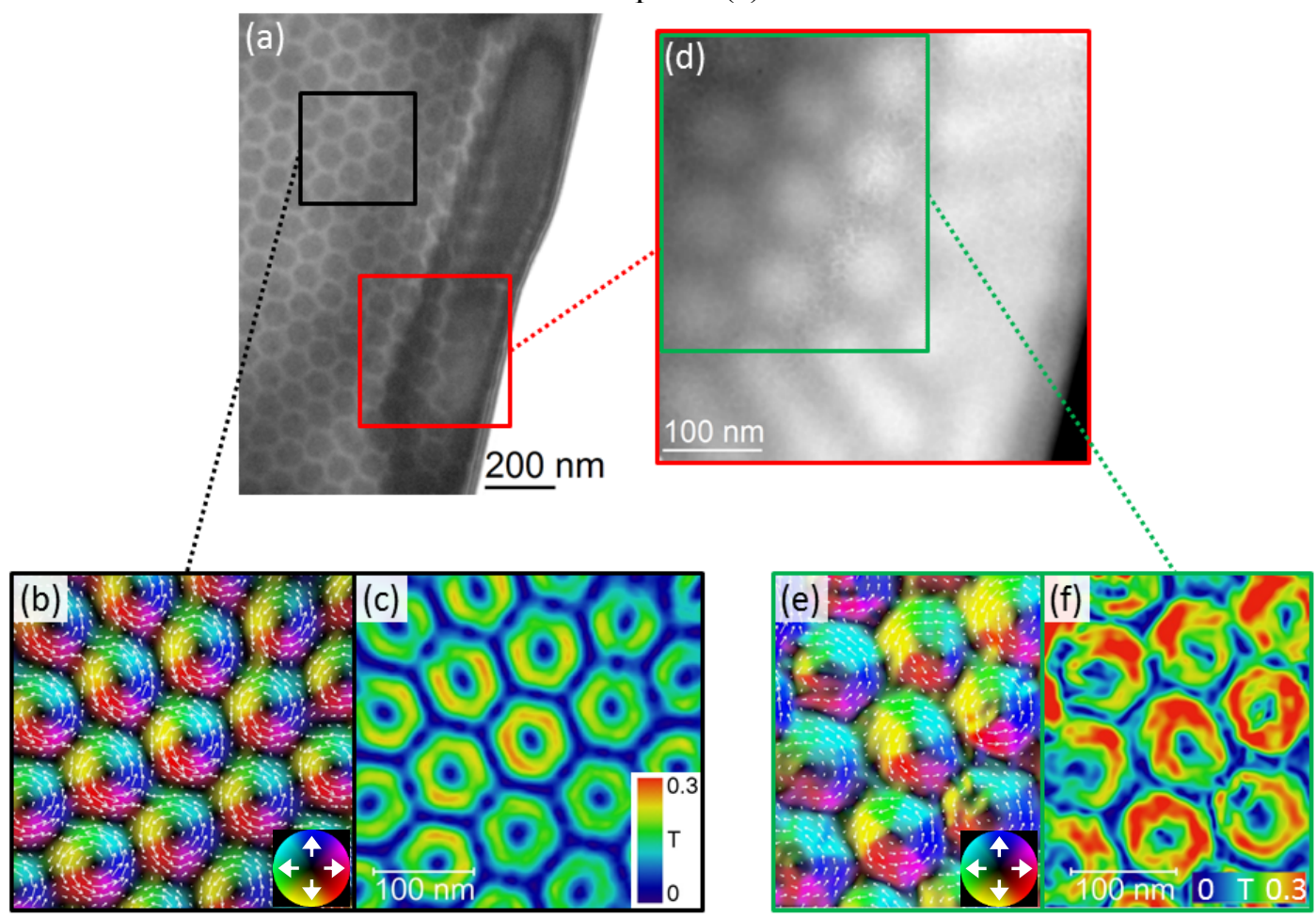

Figure 2. (a) L-TEM image in under-focus showing the skyrmions as dark contrast. (d) Phase image of the position indicated by the red square in (a). (b, e) Mapping of the direction of the in-plane magnetic flux by combining a vector plot (white arrows) and a false color image. (c, f) False colour mapping of the magnitude of the in-plane magnetic flux. 